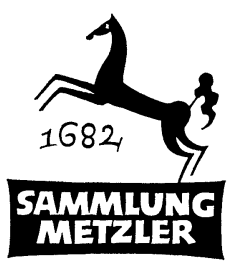

REALIEN ZUR LITERATUR

ABT. D:

LITERATURGESCHICHTE 


\section{CHARLOTTE JOLLES}

\section{Theodor Fontane}

MCMLXXII

J. B. METZLERSCHE VERLAGSBUCHHANDLUNG STUTTGART 
ISBN 978-3-476-10114-3

ISBN 978-3-476-99416-5 (eBook)

DOI 10.1007/978-3-476-99416-5

\section{114}

(C) 1972 Springer-Verlag GmbH Deutschland

Ursprünglich erschienen 1972 bei J. B. Metzlersche Verlagsbuchhandlung und Carl Ernst Poeschel Verlag GmbH in Stuttgart 


\section{INHALT}

I. Materialien . . . . . . . . . . . . . . . . 1

1. Der Handschriftliche Nachlaß. Das Theodor-FontaneArchiv . . . . . . . . . . . . . . . . . 1

2. Gesamtausgaben . . . . . . . . . . 5

3. Briefe und Tagebücher . . . . . . . . . . . 8

4. Bibliographische Hilfsmittel. Ausstellungskataloge . . 13

II. VITA . . . . . . . . . . . . . . . . 15

1. Monographien . . . . . . . . . . . . 15

2. Herkunft . . . . . . . . . . . . . . 17

3. Frühe Jahre (bis 1850) . . . . . . . . . . . 19

4. 1850-1859 . . . . . . . . . . . . . . . 26

5. $1860-1898$. . . . . . . . . . . . . . . 28

III. Das Gesamtwerk . . . . . . . . . . . . . . 32

a) Gedichte . . . . . . . . . . . . . . . . 32

b) Journalismus . . . . . . . . . . . . . . . 36

1. Frühe Publizistik (1842-1850). Publizistik über England . . . . . . . . . . . . . . 36

2. Die Wanderungsbände . . . . . . . . . . 37

3. Die Kriegsbücher . . . . . . . . . . . . 39

4. Kritik (Theater, Literatur, Kunst) . . . . . . 41

c) Romane und Novellen (in chronologischer Ordnung) 45

"Vor dem Sturm* 45 - "Grete Minde* 52 - "L'

Adultera* 54 - "Ellernklipp* 56 - "Schach von Wuthenow * 57 - "Graf Petöfy * 61 - „Unterm Birnbaum* 62 - Cécile* 64 - Irrungen, Wirrungen * 66 - »Stine* 69 - "Quitt« 70 - "Unwiederbringlich« 73 - Frau Jenny Treibel 476 - Effi Briest 78 - Die Poggenpuhls« 83 - Der Stechlin 85 - "Mathilde Möhring 94

d) Kleine Prosaarbeiten . . . . . . . . . . . . 97

e) Fragmente und Entwürfe . . . . . . . . . . 97

f) Biographisches und Autobiographisches . . . . . 99

IV. Politik und Gesellschaft - Persönlichkeit und Weltbild . . . . . . . . . . . . . . 105

V. WIRKUNGS- UND ForSChUNGSGESCHICHTE . . . . . 112

Register . . . . . . . . . . . . . . . 122 


\begin{tabular}{|c|c|}
\hline $\begin{array}{l}\text { ADB } \\
\text { ASNS }\end{array}$ & $\begin{array}{l}\text { Allgemeine Deutsche Biographie } \\
\text { Archiv für das Studium der neueren Sprachen und } \\
\text { Literaturen }\end{array}$ \\
\hline DA & Dissertation Abstracts \\
\hline $\mathrm{DR}$ & Deutsche Rundschau \\
\hline Dt. Beitr. & Deutsche Beiträge \\
\hline DU & Deutschunterricht \\
\hline DVjs. & $\begin{array}{l}\text { Deutsche Vierteljahrsschrift für Literaturwissenschaft } \\
\text { und Geistesgeschichte }\end{array}$ \\
\hline EG & Etudes Germaniques \\
\hline Euph. & Euphorion \\
\hline FAP & Fontane-Archiv, Potsdam \\
\hline FBl. & Fontane Blätter \\
\hline FMLS & Forum for Modern Language Studies \\
\hline GLL & German Life \& Letters \\
\hline GQ & German Quarterly \\
\hline GR & Germanic Review \\
\hline GRM & Germanisch-Romanische Monatsschrift \\
\hline $\mathrm{HA}$ & Hanser-Ausgabe der Werke \\
\hline Hochl. & Hochland \\
\hline Hs., Hss. & Handschrift(en) \\
\hline Jb. f. Br. Lg. & Jahrbuch für Brandenburgische Landesgeschichte \\
\hline $\begin{array}{l}\text { Jb. DSG } \\
\text { Jb. FDH }\end{array}$ & $\begin{array}{l}\text { Jahrbuch der Deutschen Schiller-Gesellschaft } \\
\text { Jahrbuch des Freien Deutschen Hochstifts }\end{array}$ \\
\hline Jb. PK & Jahrbuch Preußischer Kulturbesitz \\
\hline JEGP & Journal of English and Germanic Philology \\
\hline Mag. Lit. & Magazin für die Literatur des In- und Auslandes \\
\hline Marg. & Marginalien \\
\hline Mh. & Monatshefte (Wisconsin) \\
\hline ML & Modern Languages \\
\hline MLN & Modern Language Notes \\
\hline MLR & Modern Language Review \\
\hline Ms. & Manuskript \\
\hline MSp. & Muttersprache \\
\hline $\mathrm{NDH}$ & Neue Deutsche Hefte \\
\hline NDL & Neue Deutsche Literatur \\
\hline NR & Neue Rundschau \\
\hline NSR & Neue Schweizer Rundschau \\
\hline NyA & Nymphenburger Ausgabe der Werke \\
\hline OL & Orbis Litterarum \\
\hline PMLA & Publications of the Modern Language Association \\
\hline
\end{tabular}


RG Revue Germanique

RLV Revue des langues vivantes

SuF Sinn und Form

WB Weimarer Beiträge

Weiße Bll. Weiße Blätter

WW Wirkendes Wort

ZfBFr. Zeitschrift für Bücherfreunde

ZfBibl. Zentralblatt für Bibliothekswesen

ZfdB Zeitschrift für deutsche Bildung

ZfdPh. Zeitschrift für deutsche Philologie

ZfdU Zeitschrift für deutschen Unterricht

Zs. Zeitschrift 
Die folgenden Veröffentlichungen werden im Text oder in den Literaturangaben zu den Romanen oft nur mit Namen und Jahreszahl zitiert.

Bosshart, Adelheid: Th Fs historische Romane. 1957.

Demetz, Peter: Formen des Realismus. Th F. Kritische Untersuchungen. 1964.

Fricke, Hermann: Th. F. Chronik seines Lebens. 1960.

GüNTHER, Vincent J.: Das Symbol im erzählerischen Werk Fs. 1967.

HaYens, Kenneth: Th. F. A critical study. London 1920.

LuKács, Georg: Der alte F. In: G.L.: Deutsche Realisten des 19. Jhs. 1951, S. 262-307.

Martini, Fritz: Th. F. In: F.M.: Deutsche Literatur im bürgerlichen Realismus. 1962; ${ }^{2} 1964$, S. 737-800.

MrtTenZweI, Ingrid: Die Sprache als Thema. Untersuchungen zu Fs Gesellschaftsromanen. 1970.

OHL, Hubert: Bild und Wirklichkeit. Studien z. Romankunst Raabes u. Fs. 1968.

Pascal, Roy: Th. F. In: R. P.: The German novel. Manchester 1956, S. $178-214$.

Reuter, Hans-Heinrich: F. 2 Bde. 1968.

Rosenfeld, Hans-Friedrich: Zur Entstehung Fontanescher Romane. Groningen/Den Haag 1926.

Rost, Wolfgang E.: Ortlichkeit u. Schauplatz in Fs Werken. 1931.

Schillemeit, Jost: Th. F. Geist u. Kunst seines Alterswerks. 1961.

SIEPER, Clara: Der historische Roman u. die historische Novelle bei Raabe u. F. 1930.

WANDREY, Conrad: Th. F. 1919.

Sammelwerke, in den Literaturangaben gekürzt zitiert:

Theodor Fontanes Werk in unserer Zeit. Symposion z. 30-JahrFeier des Fontane-Archivs der Brandenburgischen Landes- $u$. Hochschulbibliothek Potsdam. 1966.

Fontanes Realismus. Wissenschaftliche Konferenz z. 150. Geb. Th. Fs in Potsdam. Vorträge u. Berichte. Im Auftr. d. Dt. Staatsbibliothek hrsg. v. Hans-Erich Teitge u. Joachim Schobeß. 1972. 
Zeitschriften und Zeitungen mit Erstveröffentlichungen Fs, im Text gekürzt zitiert:

Cosmopolis. Intern. Revue. (London, Wien, Paris.)

Daheim. Ein dt. Familienblatt, mit Illustrationen. (Leipzig.)

Das Magazin für die Literatur des In- und Auslandes. Wochenschrift d. Weltliteratur. (Berlin.)

Deutsche Rundschau. (Berlin.)

Deutschland. Wochenschrift f. Kunst, Literatur, Wissenschaft u. soziales Leben. (Glogau.)

Die Gartenlaube. Illustr. Familienblatt. (Leipzig.)

Nord und Süd. Eine dt. Monatsschrift. (Berlin.)

Pan. (Berlin.)

Über Land und Meer. Illustr. Wochenblatt. (Stuttgart.)

Universum. Illustrierter Hausschatz f. Poesie, Natur u. Welt, Literatur, Kunst u. Wissenschaft. (Dresden.)

Vom Fels zum Meer. Illustr. Zeitschr. f. d. dt. Haus (Stuttgart.)

Vossische Zeitung. Königlich privileg. Berlinische Ztng von Staats- $\mathrm{u}$. gelehrten Sachen. Vossische Zeitung. (Berlin.)

Westermanns Illustrierte Deutsche Monatshefte. (Braunschweig.)

Die Verfasserin dankt Barbara Mockert und Karin Hellmer vom Institute of Germanic Studies, Universität London, für ihre Hilfe bei der Zusammenstellung der bibliographischen Angaben. 